\title{
A SEARCH FOR MOLECULAR TRANSITIONS IN THE 22-25 GHZ BAND IN COMET KOHOUTEK $1973 f$
}

E. Churchwell, T Landecker, G. Winnewisser, R. Hills and J. Rahe

Introduction

According to current theories, the radicals optically identified in cometary spectra stem from more complex parent compounds which have no transitions at visible wavelengths. In recent years radio transitions of likely molecules have repeatedly been searched for but were never found (e.g., Huebner and Snyder, 1970; Clark et al., 1971). Only in Comet Kohoutek $1973 f$ have microwave lines of three molecules been detected, OH (Biraud et al., 1974; Turner, 1974), $\mathrm{CH}_{3} \mathrm{CN}$ (Ulich and Conklin, 1974), and HCĩ (Snyder et al., 1974). Here we report on our search for transitions in the $22-25 \mathrm{GHz}$ band of the molecules $\mathrm{H}_{2} \mathrm{O}, \mathrm{NH}_{3}$, and $\mathrm{CH}_{3} \mathrm{OH}$ in this Comet and give upper limits on line temperatures and column densities.

\section{Observations}

The observations of Comet Kohoutek $1973 \mathrm{f}$ have been made with the 100-m radio telescope of the Max-Planck-Institut für Radioastronomie in Bonn, West Germany. The antenna half-power beam width (HPBW) was $\sim 40$ arc sec and the aperture efficiency was $\sim 0.17$ (not accurately measured). The receiver was an uncooled tunnel diode mixer system with a double side band system temperature of $\sim 2500 \mathrm{~K}$. The observations we re made by beam-svritching with the signal beam on the electrical axis of the telescope and the comparison beam offset by 2 arc min; the switch rate was $1 \mathrm{cps}$. A calíbration signal from a noise diode was injected into the signal channel every second cycle. The temperature equivalent of the noise diode was meisured in the laboratory and checked periodically throughout the observations against NGC 7027. The pointing accuracy of the telescope was $\leqq 10$ arc sec. 
The observations were carried out in two periods, the first on January 7 and 8,1974 , when the Comet had passed perihelion (perihelion passage, $T^{\prime}=1973$, Dec.28.4; perihelion distance, $q=0.14$ AU) but had a high enough angular separation from the sur so that the sun was outside the beam pattern; the second on January 14 and 15, 1974, when the Comet reached its closest approach to the Earth (Jan. 15, 1974, $\Delta=0.81 \mathrm{AU}$ ), thus giving the best ratio of source size (head of the Comet) to beam size.

The essential observational data is listed in Table 1. The ephemeris was calculated by Stumpff from elements communicated by Marsden (1974). The first six columns of Table 1 list the lime of observation, position of the Comet, its heliocentric and geocentric distance, radial velocity, and the observed frequency. The total number of scans is given in column 7. Pach scan lasted for 10 minutes. Since the Comet's position was not known with high accuracy on January 7 and 8, 1974, the telescope was pointed at the presumed position of the cometary moleus and also at 4 positions surrounding it with $\alpha^{\prime}=\alpha_{0} \pm 30^{\prime \prime}$ and $\delta^{\prime}=\delta_{0} \pm 30^{\prime \prime}$, respectively (with $\alpha_{0}, \delta_{0}$ the preaicted position of the nucleus). In searching for $\mathrm{H}_{2} \mathrm{O}$ on January 7 and 8 , four scans each wert made at the presumed nucleus' position as well as at $\alpha^{\prime}=\alpha_{0}+30^{\prime \prime}$ and $\delta^{\prime}=\delta_{0}-30 "$. At each of the remaining two positions $\alpha^{\prime}=\alpha_{0}-30 "$ and $\delta^{\prime}=\delta_{0}+30^{\prime \prime}$, three scans were made. During the search for $\mathrm{iri}_{3}$ on January 8, three scans were made at the nucleus and two scans each at $\alpha^{\prime}=\alpha_{0} \pm 30 "$ and $\delta^{\prime}=\delta_{0} \pm 30^{\prime \prime}$. Since the Comet's orbit had been confirmed before the second observation period on Jan. 1.4/15 started, the telescope was pointed only at the predicted position of the nucleus. 


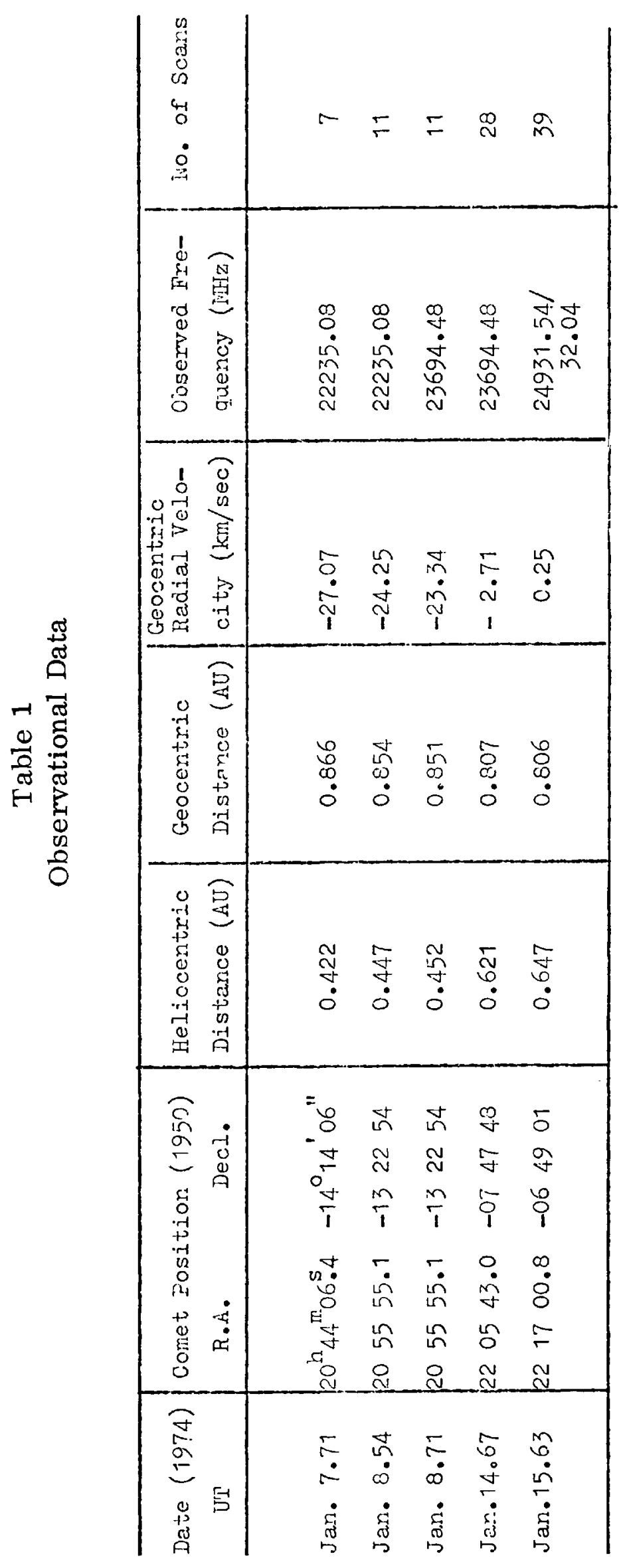


It was attempted to detect radio lines in the $1.3-\mathrm{cm}$ band of three molecular species which had been detected in interstellar space but not in a comet.

$\mathrm{NH}_{3}$ (ammonia) is chemically very stable and a comrion end product of reactions where polymers or hyörogen bonded molecules are pyrolyzed. This yrocess is expected to take place during the Comet's closest approach to the sun. Also this molecule presumably has one of the greatest extents around the nucleus of any molecule observable at this frequency. Jt may be noted that: the (1.1) transition at $23694.48 \mathrm{MHz}$ is energetically the lowest in paraammonia, whereas the (3.3) inversion transition at $23870.11 \mathrm{MHz}$ is the lowest in orth-ammonia.

At the time of the search, $\mathrm{H}_{2} \mathrm{O}$ (water) had not directly been observed in a somet. AIth ough high excitation energies are required to observe the $\mathrm{H}_{2} \mathrm{O}$ rotational transition line, the chemical stability of the molecule in conjunction with the high abundance of cometary $\mathrm{OH}$ and $\mathrm{H}$ seemed to warrant a search for this species. $\mathrm{H}_{2} \mathrm{O}$ was subsequently found in Comet Bradfield 1974b (Clark et al., 1974).

$\mathrm{CH}_{3} \mathrm{OH}$ (methyl alcohol) is a rather common molecule in the interstellar medium. The lines $(2,2-2,1 ; 3,2-3,1 ; 4,2-4,1)$ lie within a very nazrow frequency interval (24928.7 - 24934.4 $\mathrm{MHz}$ ), but they arise from energy levels (unfortunately only of species $E 1$ ) with rather different energies. Thus they are expected to be sensitive to excitation conditions. 


\section{Discussion}

No lines were detected above the noise. However, the observed noise can be used to derive an upper limit on the column densities, $\mathbb{N}_{\mathrm{m}}$ which is given by

$$
\mathrm{N}_{\mathrm{m}}=\frac{3 \mathrm{k}}{8 \pi^{3}\left|\mu_{\mathrm{ij}}\right|^{2} \nu} \int \mathrm{B} \nu \mathrm{d} \nabla
$$

Table 2 is a summary of the results. A line width $\Delta V_{L}=0.53 \mathrm{~km} / \mathrm{sec}$ was assumed which is equivalent to that found for $\mathrm{CH}_{3} \mathrm{CN}$ (Ulich and Conklin, 1974) when corrected for the half-intensity full width rather than half the $1 / e-$ width. Listed are the molecules with corresponding transition and transition frequency, and upper limit on the mean column densities.

Table 2

Results

\begin{tabular}{|c|c|c|c|c|c|c|}
\hline Molecule & Transition & $\begin{array}{c}\text { Frequency } \\
\text { (NHz) }\end{array}$ & $\Delta \mathrm{T}_{\mathrm{pp}}(\mathrm{K})$ & $\mathrm{T}_{\mathrm{A}_{\mathrm{L}}}(\mathrm{rms})(\mathrm{K})$ & $\mathrm{T}_{\mathrm{B}_{\mathrm{L}}}(3 \sigma)(\mathrm{K})$ & $N\left(\right.$ molecules $\left./ \mathrm{cm}^{2}\right)$ \\
\hline $\mathrm{H}_{2} \mathrm{O}$ & $6_{16}-5_{23}$ & 22235.08 & 1.07 & $\leq 0.2$ & $\leq 2$ & $\leq 5.6 \times 10^{14}$ \\
\hline $\mathrm{CH}_{3} \mathrm{OH}$ & $4_{2}-4_{1}$ & 24933.47 & 0.75 & $\leq 0.15$ & $\leq 1.5$ & $\leq 6.6 \times 10^{12}$ \\
\hline $\mathrm{III}_{3}$ & $(1,1)$ & 23694.48 & 0.7 & $\leq 0.14$ & $\leq 0.47$ & $\leq 1.7 \times 10^{12}$ \\
\hline
\end{tabular}




\section{REFERENCES}

Biraud, F., Bourgois, G., Crovisier, J., Fillit, R., Gerard, E., and Kazes, I., 1974, Astron. and Ap. 34, 163.

Clark, Tb A., Donn, B., Jackson, W. M., Sullivan, W. T. III, and Vandenberg, N., 1971, A. J., 76, 614.

Huebner, W. F., and Snyder, L. E., 1970, A. J., 75, 759.

Huebner, W. F., Snyder, L. E., and Buhl, D., 1974, Icarus 23, 580.

Turner, B. E., 1974, Ap. J. (Letters), 189, L137.

Ulich, B. L., and Conklin, E. K., 1974, Nature, 248, 121. 